\title{
Diagnosis of Anterior Inferior Cerebellar Artery Occlusion on Magnetic Resonance Angiography With Reference to Basiparallel Anatomic Scanning-Magnetic Resonance Imaging -Case Report-
}

\author{
Ryoichi IWATA, Kohsuke YamAshitA, Tomofumi NishiKAWA, \\ Motohiro KAJIWARA, and Tetsuya UEBA \\ Department of Neurosurgery, Kishiwada City Hospital, Kishiwada, Osaka
}

\begin{abstract}
A 67-year-old woman presented with symptomatic infarction in the territory of the anterior inferior cerebellar artery manifesting as vertigo, vomiting, and right facial weakness. Basiparallel anatomic scanning (BPAS) magnetic resonance (MR) imaging combined with MR angiography demonstrated the occluded anterior inferior cerebellar artery. Common anatomic variations and limited detection of the smaller branches on MR angiography sometimes hinder evaluation of the occluded artery. BPAS-MR imaging may have a supplementary role to MR angiography in the diagnosis of cerebellar artery occlusions.
\end{abstract}

Key words: anterior inferior cerebellar artery, infarction, magnetic resonance imaging

Received December 10, 2009; Accepted April 7, 2010

Author's present address: Ryoichi Iwata, M.D., Department of Neurosurgery, Kansai Medical University Hirakata Hospital, Hirakata, Osaka, Japan. 


\section{Introduction}

One fifth of all ischemic strokes occur in the territory of the vertebrobasilar arteries. ${ }^{5)}$ Therefore, identification of the anatomic location and extent of ischemic lesions, and evaluation of the causative vascular lesions and mechanisms of ischemia are important for appropriate management. ${ }^{1)}$ Magnetic resonance (MR) imaging provides good diagnostic accuracy of ischemia within the posterior circulation. However, MR angiography of the vertebrobasilar arteries is affected by the common anatomic variations of the vertebrobasilar arteries. ${ }^{6)} \mathrm{MR}$ images with basiparallel anatomic scanning (BPAS) can accurately depict the outer surface of the vertebrobasilar arteries within the basal cerebrospinal fluid cisterns, regardless of the internal blood flow. ${ }^{4)}$ We treated a patient with posterior circulation infarction involving the anterior inferior cerebellar artery (AICA), and discuss noninvasive diagnosis of occlusive arterial lesions using BPAS-MR imaging and MR angiography.

\section{Case Report}

A 67-year-old woman presented with vertigo, vomiting, and right facial weakness. Neurological examination on admission showed right Horner's sign, right facial palsy, diminished hearing acuity in the right ear, right limb cerebellar ataxia, and diminished pain sensation in the left face. Diffusion- and $\mathrm{T}_{2}$-weighted $\mathrm{MR}$ imaging showed acute infarction involving the right brain stem and cerebellum, which are usually supplied by the AICA (Fig. 1). MR angiography failed to show the right AICA, whereas the left AICA was clearly visualized (Fig. 2). An arterial

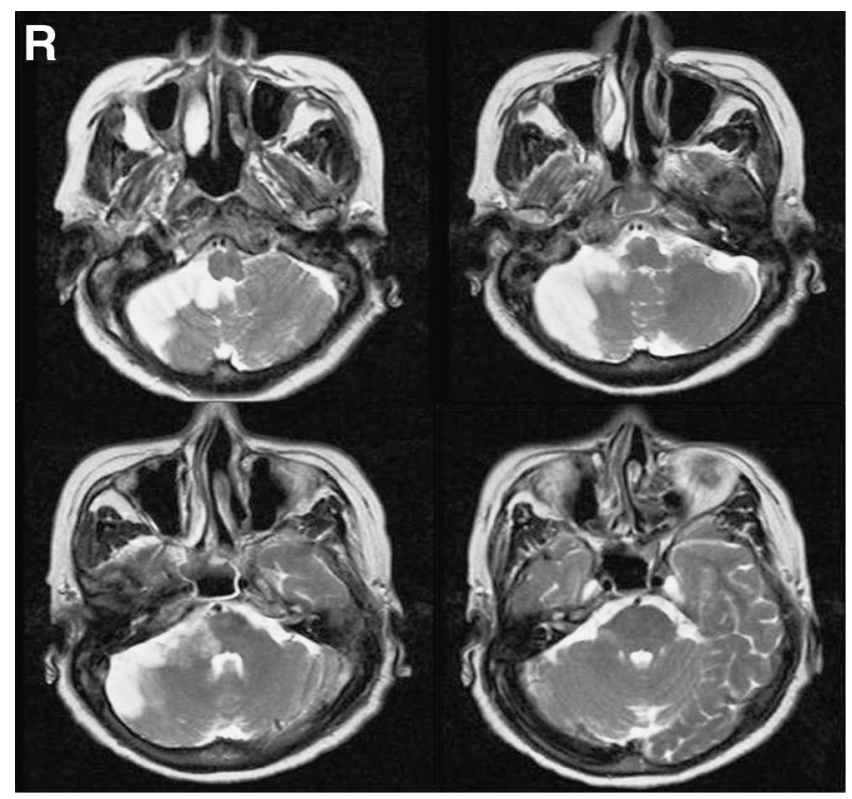

Fig. 1 Axial $\mathrm{T}_{2}$-weighted magnetic resonance images showing high intensities in the right cerebellar hemisphere, middle cerebellar peduncle, inferolateral to the right side of the pons, and medulla. stump at the presumed origin of the right AICA was found on close inspection of the MR angiograms. The right posterior inferior cerebellar artery (PICA) was not identified, so the right AICA was thought to form an AICA-PICA trunk. BPAS-MR imaging showed the right AICA as thick and tortuous like the left AICA (Fig. 3). BPAS-MR imaging (1.5 T, Gyroscan Intera; Philips, Best, the Netherlands) investigated a $20-\mathrm{mm}$ thick coronal section parallel to the clivus using fast spin-echo sequence (repetition time/echo time/excitations, $889 \mathrm{msec} / 23 \mathrm{msec} / 2 ; 150 \times 150 \mathrm{~mm}$ field of view; $136 \times 512$ matrix; TA $168 \mathrm{sec}$ ). The patient declined cerebral angiography, so radiological diagnosis of the AICA occlusion was made. Despite treatment, her neurological conditions remained almost identical to those on admission, and she was transferred to a rehabilitation facility.

\section{Discussion}

Only a few studies have evaluated MR angiography for the detection and characterization of intracranial ver-

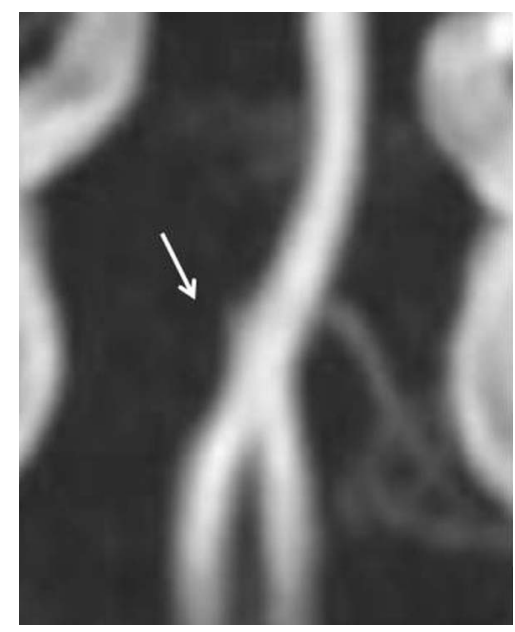

Fig. 2 Magnetic resonance angiogram showing patency of both vertebral arteries and the basilar artery, but right anterior inferior cerebellar artery (AICA) was not well visualized (arrow) despite the patency of the left AICA.

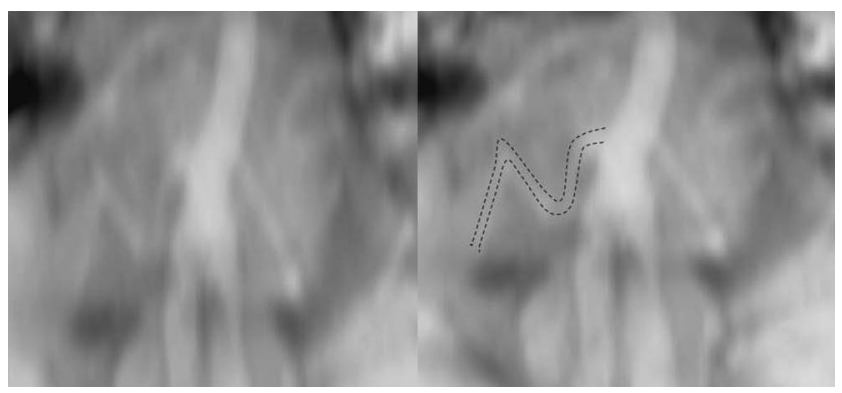

Fig. 3 Basiparallel anatomic scanning magnetic resonance images showing the normal right anterior inferior cerebellar artery (dotted line). 
tebrobasilar occlusive disease, ${ }^{6}$ and have proposed two limitations of MR angiography. The first problem is related to the spatial resolution of images, but higher magnetic field and contrast material may improve the quality of images. The second problem is related to diagnostic accuracy, as anatomic variations of the normal vertebrobasilar arteries cannot be differentiated from disease processes. ${ }^{4)}$ Indeed, apparent narrowing or absence of an artery on MR angiography may be emphasized by stenosis or occlusion, or hypoplasia or aplasia of the relevant artery. However, these considerations are the same for cerebral angiography, which is still the gold standard for evaluation of occlusive vessels. Accurate anatomic images depicting vascular structures regardless of blood flow might be useful as a guide or reference to the normal anatomy. In the present patient, BPAS-MR imaging was used for that purpose. Although the AICA was not seen on MR angiography, the presence of the AICA on BPAS-MR imaging led to a straightforward diagnosis of AICA occlusion.

BPAS-MR imaging is analogous to surface anatomic scanning, which is also based on $\mathrm{T}_{2}$-weighted imaging, and can be expected to provide an accurate three-dimensional anatomic view of the vertebrobasilar arteries. ${ }^{4)}$ Only BPAS-MR imaging has been used to depict vascular lesions with morphological changes, such as aneurysms, aplasia, and hypoplasia of the vertebrobasilar arteries. ${ }^{2,4)}$ Stenosis can be indicated by the presence of the vascular structure on BPAS-MR imaging and thinner blood flow on MR angiography. Similarly, occlusion can be identified by the presence of the vascular structure without blood flow as in the present case. These findings are very simple, straightforward, and pathognomonic.

Since MR angiography of the vertebrobasilar arteries may be misleading, reference to the anatomically accurate MR images may be important. ${ }^{2,4)}$ A stump of the occluded vertebral artery has been successfully separated from a basilar aneurysm with MR imaging using three-dimensional constructive interference in steady state sequences. ${ }^{3)}$ Arterial occlusions and hypoplastic variants of the vertebrobasilar arteries have been successfully differentiated with MR imaging using true fast imaging in steady state precession sequences. ${ }^{1}$ The present case indicates that BPAS-MR imaging may have a supplementary role to MR angiography in the diagnosis of cerebellar artery occlusions.

\section{References}

1) Freund W, Kassubek J, Aschoff AJ, Huber R: MRI-based separation of congenital and acquired vertebrobasilar artery anomalies in ischemic stroke of the posterior circulation. Stroke 39: 2382-2384, 2008

2) Hamaguchi $\mathrm{T}$, Yamada $\mathrm{M}$ : Basiparallel anatomic scanningmagnetic resonance imaging in vertebral artery dissection. Arch Neurol 66: 276-277, 2009

3) Komiyama M, Ishiguro T, Morikawa T, Nishikawa M, Yasui $\mathrm{T}$ : Distal stump of an occluded intracranial vertebral artery at the vertebrobasilar junction mimicking a basilar artery aneurysm. Acta Neurochir (Wien) 143: 1013-1017, 2001

4) Nagahata M, Abe Y, Ono S, Hosoya T, Uno S: Surface appearance of the vertebrobasilar artery revealed on basiparallel anatomic scanning (BPAS)-MR imaging: its role for brain MR examination. AJNR Am J Neuroradiol 26: 2508-2513, 2005

5) Savitz SI, Caplan LR: Vertebrobasilar disease. N Engl J Med 352: 2618-2626, 2005

6) Wentz KU, Röther J, Schwartz A, Mattle HP, Suchalla R, Edelman RR: Intracranial vertebrobasilar system: MR angiography. Radiology 190: 105-110, 1994

Address reprint requests to: Ryoichi Iwata, M.D., Department of Neurosurgery, Kansai Medical University Hirakata Hospital, 2-3-1 Shin-machi, Hirakata, Osaka 573-1191, Japan.

e-mail: ryoichi_iwata0610@yahoo.co.jp 